\title{
PRESCRIBING GAUSSIAN CURVATURE ON CLOSED RIEMANN SURFACE WITH CONICAL SINGULARITY IN THE NEGATIVE CASE
}

\author{
Yunyan Yang and Xiaobao Zhu \\ Renmin University of China, Department of Mathematics \\ Beijing 100872, P. R. China; yunyanyang@ruc.edu.cn \\ Renmin University of China, Department of Mathematics \\ Beijing 100872, P. R. China; zhuxiaobao@ruc.edu.cn
}

\begin{abstract}
The problem of prescribing Gaussian curvature on Riemann surface with conical singularities is considered. Let $(\Sigma, \beta)$ be a closed Riemann surface with a divisor $\beta$, and $K_{\lambda}=K+\lambda$, where $K: \Sigma \rightarrow \mathbf{R}$ is a Hölder continuous function satisfying $\max _{\Sigma} K=0, K \not \equiv 0$, and $\lambda \in \mathbf{R}$. If the Euler characteristic $\chi(\Sigma, \beta)$ is negative, then by a variational method, it is proved that there exists a constant $\lambda^{*}>0$ such that for any $\lambda \leq 0$, there is a unique conformal metric with the Gaussian curvature $K_{\lambda}$; for any $\lambda, 0<\lambda<\lambda^{*}$, there are at least two conformal metrics having $K_{\lambda}$ its Gaussian curvature; for $\lambda=\lambda^{*}$, there is at least one conformal metric with the Gaussian curvature $K_{\lambda^{*}}$; for any $\lambda>\lambda^{*}$, there is no certain conformal metric having $K_{\lambda}$ its Gaussian curvature. This result is an analog of that of Ding and Liu [16], partly resembles that of Borer, Galimberti and Struwe [5], and generalizes that of Troyanov [28] in the negative case.
\end{abstract}

\section{Introduction}

The problem of prescribing Gaussian curvature on smooth Riemann surfaces was proposed in [21]. Let $(\Sigma, g)$ be a closed smooth Riemann surface, $\chi(\Sigma)$ be its topological Euler characteristic, and $\kappa: \Sigma \rightarrow \mathbf{R}$ be its Gaussian curvature. If $\bar{g}=e^{2 u} g$ with a smooth function $u$, then the Gaussian curvature of $(\Sigma, \bar{g})$ satisfies $\bar{\kappa}=e^{-2 u}\left(\kappa+\Delta_{g} u\right)$, where $\Delta_{g}$ denotes the Laplace-Beltrami operator with respect to the metric $g$. A natural question is whether for any smooth function $K: \Sigma \rightarrow \mathbf{R}$, there is a smooth function $u$ such that the metric $e^{2 u} g$ has $K$ its Gaussian curvature. Clearly this is equivalent to solve the elliptic equation

$$
\Delta_{g} u+\kappa-K e^{2 u}=0 \quad \text { on } \Sigma \text {. }
$$

The Gauss-Bonnet formula leads to

$$
\int_{\Sigma} K e^{2 u} d v_{g}=\int_{\Sigma} \kappa d v_{g}=2 \pi \chi(\Sigma) .
$$

Note that the solvability of $(1)$ is closely related to the sign of $\chi(\Sigma)$. If $\chi(\Sigma)>0$, then $\Sigma$ is either the projective space $\mathbf{R} \mathbf{P}^{2}$ or the 2 -sphere $\mathbf{S}^{2}$. In the case of $\mathbf{R P}^{2}$, it was shown by Moser [24] that the equation (1) has a solution $u$, provided that $K \in C^{\infty}\left(\mathbf{S}^{2}\right)$ satisfies $\sup _{\Sigma} K>0$ and $K(p)=K(-p)$ for all $p \in \mathbf{S}^{2}$. While the problem on $\mathbf{S}^{2}$ is much more complicated and known as the Nirenberg problem, see for examples $[21,6,7,8,9]$. If $\chi(\Sigma)=0$, the problem has been completely solved by Kazdan-Warner [21]. While if $\chi(\Sigma)<0$, the problem was studied by Kazdan and Warner [21] via the method of upper and lower solutions. They proved that if $K \leq 0$

https://doi.org/10.5186/aasfm.2019.4411

2010 Mathematics Subject Classification: Primary 58E30, 53C20.

Key words: Prescribing Gaussian curvature, conical singularity. 
and $K \not \equiv 0$, then (1) has a unique solution. Later, Ding and Liu [16] considered the case that $K$ changes sign. Precisely, replacing $K$ by $K+\lambda$ in (1) with $K \leq 0, K \not \equiv 0$, and $\lambda \in \mathbf{R}$, they obtained the following conclusion by using a method of upper and lower solutions and a variational method: there exists a $\lambda^{*}>0$ such that if $\lambda \leq 0$, then (1) has a unique solution; if $0<\lambda<\lambda^{*}$, then (1) has at least two solutions; if $\lambda=\lambda^{*}$, then (1) has at least one solution; if $\lambda>\lambda^{*}$, then (1) has no solution. We refer to Aubin and Bismuth [3, 4], who gave sufficient conditions relating to $\lambda$ and $\inf _{\Sigma} K$ for solving equation (1) with $K+\lambda$. Recently, using a monotonicity technique due to Struwe [26, 27], Borer, Galimberti, and Struwe [5] partly reproved the above results and obtained additional estimates for certain sequence of solutions that allow them to characterize their bubbling behavior. Further analysis in this direction has been done by Galimberti [18], del Pino and Román [15].

The problem of prescribing Gaussian curvature can also be proposed on surfaces with conical singularities. Let $\Sigma$ be a closed Riemann surface, $p_{1}, \ldots, p_{\ell}$ be points of $\Sigma$ and $\theta_{1}, \ldots, \theta_{\ell}$ be positive numbers. Denote

$$
\bar{\chi}=2 \pi \chi(\Sigma)+\sum_{i=1}^{\ell}\left(\theta_{i}-2 \pi\right) .
$$

Then it was proved by Troyanov [28] that if $0<\bar{\chi}<\min \left\{4 \pi, 2 \theta_{1}, \ldots, 2 \theta_{\ell}\right\}$, then any smooth function on $\Sigma$, which is positive at some point is the Gaussian curvature of a conformal metric having at $p_{i}$ a conical singularity of angle $\theta_{i}$; if $\bar{\chi}=0$, then a smooth nonconstant function $K: \Sigma \rightarrow \mathbf{R}$ is the Gaussian curvature of a conformal metric having at $p_{i}$ a conical singularity of angle $\theta_{i}$ if and only if $\int_{\Sigma} K d \mu<0$, where $d \mu$ is the area element of the original singular metric; if $\bar{\chi}<0$, then any smooth negative function on $\Sigma$ is the Gaussian curvature of a unique conformal metric having at $p_{i}$ a conical singularity of angle $\theta_{i}$. As in the smooth Riemann surface case, the prescribing Gaussian curvature problem on the 2-sphere with conical singularity is most delicate. The case $\ell=2$ was studied by Chen and Li $[10,11]$. While the case $\ell \geq 3$ was considered by Eremenko [17], Malchiodi and Ruiz [23], Chen and Lin [12], De Marchis and López-Soriano [14], and others.

In this paper, we focus on the negative case, namely $\bar{\chi}<0$. Precisely we shall prove an analog of the result of Ding and Liu [16], and thereby part of results of Borer, Galimberti, and Struwe [5]. Though we still use the variational method, which had been employed by Ding and Liu, we have to overcome difficulties in the presence of conical singularities. In particular, we have to establish the strong maximum principle, which is essential for the method of upper and lower solutions in our setting.

The remaining part of this paper is organized as follows: In Section 2, we give some notations for surfaces with conical singularities and state our main results; In Section 3, the maximum principle for the Laplace-Beltrami operator and the PalaisSmale condition for certain functional are discussed; In Section 4, following the lines of $[16,5]$, we prove our main theorem.

\section{Notations and main results}

Let us briefly recall some geometric concepts from Troyanov [28]. In general, a closed Riemann surface $\Sigma$ is defined to be a topological space with an atlas $\left\{\phi_{i}: U_{i} \rightarrow\right.$ C \}, where if $U_{i} \cap U_{j} \neq \varnothing$, then the coordinate transformation $\phi_{i} \circ \phi_{j}^{-1}$ is conformal, i.e., holomorphic or anti-holomorphic. Two such atlases define the same structure on $\Sigma$ if their union is still such an atlas. A divisor on a Riemann surface is a formal sum $\beta=\sum_{i=1}^{\ell} \beta_{i} p_{i}$, where $p_{i} \in \Sigma$ and $\beta_{i}>-1, i=1, \ldots, \ell$. The set $\operatorname{supp} \beta=\left\{p_{1}, \ldots, p_{\ell}\right\}$ 
is the support of $\beta$, and the number $|\beta|=\sum_{i=1}^{\ell} \beta_{i}$ is the degree of the divisor. A conformal metric $g$ on $\Sigma$ is said to represent the divisor $\beta$ if $g \in C^{2}(\Sigma \backslash \operatorname{supp} \beta)$ verifying that if $z_{i}$ is a coordinate defined in a neighborhood $U_{i}$ of $p_{i}$, then there is some $u_{i} \in C^{2}\left(U_{i} \backslash\left\{p_{i}\right\}\right) \cap C^{0}\left(U_{i} \backslash\left\{p_{i}\right\}\right)$ such that

$$
g=e^{2 u_{i}}\left|z_{i}-z_{i}\left(p_{i}\right)\right|^{2 \beta_{i}}\left|d z_{i}\right|^{2} .
$$

Under this setting, $g$ is said to have a conical singularity of order $\beta_{i}$ or angle $\theta_{i}=$ $2 \pi\left(\beta_{i}+1\right)$ at $p_{i}, i=1, \ldots, \ell$. The Euler characteristic of $(\Sigma, \beta)$ is defined by

$$
\chi(\Sigma, \beta)=\chi(\Sigma)+|\beta|,
$$

where $\chi(\Sigma)$ is the topological Euler characteristic of $\Sigma$, and $|\beta|=\sum_{i=1}^{\ell} \beta_{i}$ is the degree of $\beta$. Let $\kappa: \Sigma \backslash \operatorname{supp} \beta \rightarrow \mathbf{R}$ be the Gaussian curvature of $g$. If $\kappa$ can be extended to a Hölder continuous function on $\Sigma$, then it was shown by Troyanov [28] that a Gauss-Bonnet formula holds:

$$
\int_{\Sigma} \kappa d v_{g}=2 \pi \chi(\Sigma, \beta)
$$

where $d v_{g}$ denotes the Riemannian volume element with respect to the conical metric $g$.

Let $(\Sigma, \beta)$ be a closed Riemann surface with a divisor $\beta=\sum_{i=1}^{\ell} \beta_{i} p_{i}$, and the metric $g$ represents $\beta$ with $\beta_{i}>-1, i=1, \ldots, \ell$. It follows from (2) that there exists a smooth Riemannian metric $g_{0}$ such that

$$
g=\rho g_{0},
$$

where $\rho>0$ on $\Sigma, \rho \in C_{\text {loc }}^{2}(\Sigma \backslash \operatorname{supp} \beta)$, and $\rho \in L^{r}(\Sigma)$ for some $r>1$. Let $W^{1,2}(\Sigma, g)$ be the completion of $C^{\infty}(\Sigma)$ under the norm

$$
\|u\|_{W^{1,2}(\Sigma, g)}=\left(\int_{\Sigma}\left(\left|\nabla_{g} u\right|^{2}+u^{2}\right) d v_{g}\right)^{1 / 2},
$$

where $\nabla_{g}$ denotes the gradient operator with respect to the metric $g$. It was observed by Troyanov in $[28]$ that $W^{1,2}(\Sigma, g)=W^{1,2}\left(\Sigma, g_{0}\right)$. As a consequence, by the Sobolev embedding theorem for smooth Riemann surface $\left(\Sigma, g_{0}\right)$ and the Hölder inequality, one has

$$
W^{1,2}(\Sigma, g) \hookrightarrow L^{p}(\Sigma, g), \quad \forall p>1 .
$$

We now state the following:

Theorem 1. Let $(\Sigma, \beta)$ be a closed Riemann surface with a divisor $\beta=\sum_{i=1}^{\ell} \beta_{i} p_{i}$. Suppose that the Euler characteristic $\chi(\Sigma, \beta)<0, K: \Sigma \rightarrow \mathbf{R}$ is a Hölder continuous function, $\max _{\Sigma} K=0$ and $K \not \equiv 0$. Let $K_{\lambda}=K+\lambda, \lambda \in \mathbf{R}$. Assume that a conformal metric $g$ represents $\beta$. Let $\kappa: \Sigma \backslash \operatorname{supp} \beta \rightarrow \mathbf{R}$ be the Gaussian curvature of $g$, and $\kappa$ can be extended to a Hölder continuous function on $\Sigma$. Then there exists a constant $\lambda^{*}>0$ such that

(i) when $\lambda \leq 0$, there exists a unique conformal metric on $\Sigma$ with Gaussian curvature $K_{\lambda}$, representing the divisor $\beta$;

(ii) when $0<\lambda<\lambda^{*}$, there exist at least two conformal metrics on $\Sigma$ with the same Gaussian curvature $K_{\lambda}$, representing the divisor $\beta$;

(iii) when $\lambda=\lambda^{*}$, there exists at least one conformal metric on $\Sigma$ with Gaussian curvature $K_{\lambda^{*}}$, representing the divisor $\beta$; (iv) when $\lambda>\lambda^{*}$, there is no function $u \in W^{1,2}(\Sigma, g) \cap C^{2}(\Sigma \backslash \operatorname{supp} \beta) \cap C^{0}(\Sigma)$ such that $e^{2 u} g$ has the Gaussian curvature $K_{\lambda}$. 
Since the metric $g$ has the Gaussian curvature $\kappa$, and the metric $g_{\lambda}=e^{2 u} g$ has the Gaussian curvature $K_{\lambda}=K+\lambda$. A standard calculation shows

$$
\Delta_{g} u+\kappa-K_{\lambda} e^{2 u}=0 \quad \text { on } \Sigma \backslash \operatorname{supp} \beta .
$$

Note that if $u \in W^{1,2}(\Sigma, g)$ is a distributional solution of the equation

$$
\Delta_{g} u+\kappa-K_{\lambda} e^{2 u}=0 \text { on } \Sigma,
$$

we have by elliptic estimates $u \in C^{2}(\Sigma \backslash \operatorname{supp} \beta) \cap C^{0}(\Sigma)$, and thus (6) holds. Hence, in order to prove Theorem 1, it suffices to show the following:

Theorem 2. Under the same assumptions as in Theorem 1, there exists a $\lambda^{*}>0$ such that

(i) if $\lambda \leq 0$, then (7) has a unique distributional solution;

(ii) if $0<\lambda<\lambda^{*}$, then (7) has at least two distributional solutions;

(iii) if $\lambda=\lambda^{*}$, then (7) has at least one distributional solution;

(iv) if $\lambda>\lambda^{*}$, then (7) has no distributional solution.

For the proof of Theorem 2, we follow closely Ding and Liu [16] by employing a variational method. In particular we use the upper and lower solutions principle and the strong maximum principle. In the remaining part of this paper, $(\Sigma, g)$ will always denote a conical singular Riemann surface given in Theorem 1; we do not distinguish sequence and subsequence; moreover we often denote various constants by the same $C$, even in the same line.

\section{Preliminary analysis}

In this section, we prove maximum principle, Palais-Smale condition, upper and lower solutions principle, which will be used later. Compared with the smooth Riemann surface case, all the above mentioned things need to be re-established since the metric $g$ has conical singularities.

3.1. Maximum principle. We first have a weak maximum principle by integration by parts, namely

Lemma 3. (Weak maximum principle) For any constant $c>0$, if $u \in W^{1,2}(\Sigma, g)$ $\cap C^{0}(\Sigma)$ satisfies $\Delta_{g} u+c u \geq 0$ in the distributional sense, then $u \geq 0$ on $\Sigma$.

Proof. Denote $u^{-}=\min \{u, 0\}$. Testing the equation $\Delta_{g} u+c u \geq 0$ by $u^{-}$, one has

$$
\int_{\Sigma}\left(\left|\nabla_{g} u^{-}\right|^{2}+c u^{-2}\right) d v_{g} \leq 0
$$

This leads to $u^{-} \equiv 0$ on $\Sigma$.

Moreover, using the Moser iteration (see for example Theorems 8.17 and 8.18 in [19]), we obtain the following strong maximum principle.

Lemma 4. (Strong maximum principle) Let $u \in W^{1,2}(\Sigma, g) \cap C^{0}(\Sigma)$ satisfy that $u \geq 0$ on $\Sigma$, and that for some positive constant $c, \Delta_{g} u+c u \geq 0$ in the distributional sense. If there exists a point $x_{0} \in \Sigma$ such that $u\left(x_{0}\right)=0$, then there holds $u \equiv 0$ on $\Sigma$.

Proof. Step 1. If $v \in W^{1,2}(\Sigma, g) \cap C^{0}(\Sigma)$ satisfies $v \geq 0$ on $\Sigma$, and

$$
\Delta_{g} v-c v \leq 0
$$


in the distributional sense, where $c$ is a positive constant, then there exists some constant $C$ depending only on $(\Sigma, g)$ such that

$$
\|v\|_{L^{\infty}(\Sigma)} \leq C\|v\|_{L^{2}(\Sigma, g)} .
$$

Now we use the Moser iteration to prove (9). For any $p \geq 2$, testing (8) by $v^{p-1}$ and integrating by parts, we have

$$
\int_{\Sigma}\left|\nabla_{g} v^{\frac{p}{2}}\right|^{2} d v_{g} \leq \frac{c p^{2}}{4(p-1)} \int_{\Sigma} v^{p} d v_{g}
$$

Hence $\left\|v^{\frac{p}{2}}\right\|_{W^{1,2}(\Sigma, g)} \leq C p\left\|v^{\frac{p}{2}}\right\|_{L^{2}(\Sigma, g)}$ for some constant $C$. Then the Sobolev embedding (5) leads to $\left\|v^{\frac{p}{2}}\right\|_{L^{4}(\Sigma, g)} \leq C p\left\|v^{\frac{p}{2}}\right\|_{L^{2}(\Sigma, g)}$, which is equivalent to $\|v\|_{L^{2 p}(\Sigma, g)} \leq$ $C^{\frac{2}{p}} p^{\frac{2}{p}}\|v\|_{L^{p}(\Sigma, g)}$. Taking $p=p_{k}=2^{k}, k=1,2, \ldots$, we have

$$
\begin{aligned}
& \|v\|_{L^{p_{k+1}}(\Sigma, g)} \leq C^{\frac{2}{p_{k}}} p_{k}^{\frac{2}{p_{k}}}\|v\|_{L^{p_{k}}(\Sigma, g)} \leq C^{\sum_{j=1}^{k} 2^{1-j}} 2^{\sum_{j=1}^{k} 2^{1-j} j}\|v\|_{L^{2}(\Sigma, g)} \\
& \leq C\|v\|_{L^{2}(\Sigma, g)} \text {. }
\end{aligned}
$$

Letting $k \rightarrow \infty$ in (10), we conclude (9).

Step 2. Let $u \in W^{1,2}(\Sigma, g) \cap C^{0}(\Sigma)$ be a nonnegative distributional solution of

$$
\Delta_{g} u+c u \geq 0
$$

where $c$ is a positive constant. Then there exists some constant $C$ such that

$$
\|u\|_{L^{2}(\Sigma, g)} \leq C \inf _{\Sigma} u \text {. }
$$

Without loss of generality, we assume $u \geq \epsilon>0$, otherwise we can replace $u$ by $u+\epsilon$. We claim that that $u^{-1}$ is a distributional solution of $\Delta_{g} u^{-1}-c u^{-1} \leq 0$. To see it, we recall that $g=\rho g_{0}$, where $\rho: \Sigma \rightarrow \mathbf{R}$ is a positive function, $\rho \in L^{q}(\Sigma)$ for some $q>1$, and $g_{0}$ is a smooth Riemannian metric. Then for any $\phi \in W^{1,2}\left(\Sigma, g_{0}\right)$ with $\phi \geq 0$, we calculate

$$
\begin{aligned}
& \int_{\Sigma}\left(\nabla_{g} u^{-1} \nabla_{g} \phi-c u^{-1} \phi\right) d v_{g}=\int_{\Sigma}\left(\nabla_{g_{0}} u^{-1} \nabla_{g_{0}} \phi-c \rho u^{-1} \phi\right) d v_{g_{0}} \\
& =-\int_{\Sigma}\left(\nabla_{g_{0}} u \nabla_{g_{0}}\left(\phi u^{-2}\right)+2 \phi u^{-3}\left|\nabla_{g_{0}} u\right|^{2}+c \rho u\left(\phi u^{-2}\right)\right) d v_{g_{0}} \\
& \leq-\int_{\Sigma}\left(\nabla_{g_{0}} u \nabla_{g_{0}}\left(\phi u^{-2}\right)+c \rho u\left(\phi u^{-2}\right)\right) d v_{g_{0}} \\
& =-\int_{\Sigma}\left(\nabla_{g} u \nabla_{g}\left(\phi u^{-2}\right)+c u\left(\phi u^{-2}\right)\right) d v_{g} .
\end{aligned}
$$

This together with (11) confirms our claim. Now we have by Step 1,

$$
\sup _{\Sigma} u^{-1} \leq C\left\|u^{-1}\right\|_{L^{2}(\Sigma, g)}
$$

which implies that

$$
\inf _{\Sigma} u \geq C\left(\int_{\Sigma} u^{-2} d v_{g}\right)^{-\frac{1}{2}}=C\left(\int_{\Sigma} u^{-2} d v_{g} \int_{\Sigma} u^{2} d v_{g}\right)^{-1 / 2}\left(\int_{\Sigma} u^{2} d v_{g}\right)^{1 / 2} .
$$

Thus, to prove (12), it suffices to show that there exists some constant $C$ such that

$$
\int_{\Sigma} u^{-2} d v_{g} \int_{\Sigma} u^{2} d v_{g} \leq C
$$


Let $w=\log u-\gamma$, where $\gamma=\frac{1}{\operatorname{Vol}_{g}(\Sigma)} \int_{\Sigma} \log u d v_{g}$. We shall prove that

$$
\int_{\Sigma} e^{2|w|} d v_{g} \leq C
$$

which implies

$$
\int_{\Sigma} e^{2(\gamma-\log u)} d v_{g} \leq C, \quad \int_{\Sigma} e^{2(\log u-\gamma)} d v_{g} \leq C .
$$

This immediately leads to (13).

We are only left to prove (14). Testing the equation (11) by $u^{-1}$, we have

$$
\int_{\Sigma}\left(\nabla_{g} u^{-1} \nabla_{g} u+c\right) d v_{g} \geq 0 .
$$

It follows that

$$
\int_{\Sigma}\left|\nabla_{g} w\right|^{2} d v_{g} \leq C
$$

Note that $\int_{\Sigma} w d v_{g}=0$. In view of $(15)$, we conclude from the Poincaré inequality that

$$
\|w\|_{W^{1,2}(\Sigma, g)} \leq C .
$$

Recall that the metric $g$ represents the divisor $\beta=\sum_{i=1}^{\ell} \beta_{i} p_{i}$ with $\beta_{i}>-1, i=$ $1, \ldots, \ell$. Denote $b=\min \left\{1,1+\beta_{1}, \ldots, 1+\beta_{\ell}\right\}$. Then the Trudinger-Moser inequality for surfaces with conical singularities [28] together with (16) implies that

$$
\int_{\Sigma} e^{2|w|} d v_{g} \leq \int_{\Sigma} e^{\frac{b w^{2}}{\|w\|_{W^{1,2}(\Sigma, g)}^{2}}+\frac{1}{b}\|w\|_{W^{1,2(\Sigma, g)}}^{2}} d v_{g} \leq C \int_{\Sigma} e^{\frac{b w^{2}}{\|w\|_{W^{1,2}(\Sigma, g)}^{2}}} d v_{g} \leq C .
$$

Thus (14) holds and the proof of Step 2 terminates.

One can easily see that the conclusion of the lemma follows from (12).

It is remarkable that only subcritical Trudinger-Moser inequality was employed in (17). Such inequalities are important tools in geometry and analysis. For more details, we refer the reader to recent works $[1,22,25,29,30,13,20]$ and the references therein.

3.2. Palais-Smale condition. For any $\lambda \in \mathbf{R}$, we define a functional $E_{\lambda}$ : $W^{1,2}(\Sigma, g) \rightarrow \mathbf{R}$ by

$$
E_{\lambda}(u)=\int_{\Sigma}\left(\left|\nabla_{g} u\right|^{2}+2 \kappa u-K_{\lambda} e^{2 u}\right) d v_{g}
$$

where $\kappa: \Sigma \rightarrow \mathbf{R}$ is the Gaussian curvature of $g, K_{\lambda}=K+\lambda$ is defined as in Theorem 1.

Lemma 5. (Palais-Smale condition) Suppose that $\Sigma_{\lambda}^{-}=\left\{x \in \Sigma: K_{\lambda}<0\right\}$ is nonempty for some $\lambda \in \mathbf{R}$. Then $E_{\lambda}$ satisfies the $(P S)_{c}$ condition for all $c \in \mathbf{R}$, i.e., if $u_{j}$ is a sequence of functions in $W^{1,2}(\Sigma, g)$ such that $E_{\lambda}\left(u_{j}\right) \rightarrow c$ and $d E_{\lambda}\left(u_{j}\right) \rightarrow 0$, then there exists some $u_{0} \in W^{1,2}(\Sigma, g)$ satisfying $u_{j} \rightarrow u_{0}$ in $W^{1,2}(\Sigma, g)$.

Proof. Let $\left(u_{j}\right)$ be a function sequence such that $E_{\lambda}\left(u_{j}\right) \rightarrow c$ and $d E_{\lambda}\left(u_{j}\right) \rightarrow 0$, or equivalently

$$
\begin{aligned}
& \int_{\Sigma}\left(\left|\nabla_{g} u_{j}\right|^{2}+2 \kappa u_{j}-K_{\lambda} e^{2 u_{j}}\right) d v_{g}=c+o_{j}(1), \\
& \int_{\Sigma}\left(\nabla_{g} u_{j} \nabla_{g} \varphi+\kappa \varphi-K_{\lambda} e^{2 u_{j}} \varphi\right) d v_{g}=o_{j}(1)\|\varphi\|_{W^{1,2}(\Sigma, g)}, \quad \forall \varphi \in W^{1,2}(\Sigma, g),
\end{aligned}
$$


where $o_{j}(1) \rightarrow 0$ as $j \rightarrow \infty$.

Note that $\operatorname{supp} \beta=\left\{p_{1}, \ldots, p_{\ell}\right\}$ is a set of finite points. $\Sigma_{\lambda}^{-} \backslash \operatorname{supp} \beta$ must contain a domain $\Omega$ such that the closure of $\Omega$ is also contained in $\Sigma_{\lambda}^{-} \backslash \operatorname{supp} \beta$. In view of (4), there would exist two positive constants $C_{1}$ and $C_{2}$ depending only on $\Omega$ such that

$$
C_{1} g_{0} \leq g \leq C_{2} g_{0} \quad \text { on } \quad \Omega \text {. }
$$

Denote $u_{j}^{+}=\max \left\{u_{j}, 0\right\}$. Based on an argument of Ding and Liu ([16], Lemma 2), where a mistake was corrected by Borer, Galimberti and Struwe ([5], Appendix), for another domain $\Omega^{\prime} \subset \subset \Omega$, there exists a positive constant $C$ depending only on $C_{1}$, $C_{2}$ and $\operatorname{dist}_{g}\left(\Omega^{\prime}, \partial \Omega\right)$ such that

$$
\int_{\Omega^{\prime}}\left(\left|\nabla_{g} u_{j}^{+}\right|^{2}+u_{j}^{+2}\right) d v_{g} \leq C .
$$

Taking $\varphi \equiv 1$ in $(20)$, one has

$$
\int_{\Sigma} K_{\lambda} e^{2 u_{j}} d v_{g}-\int_{\Sigma} \kappa d v_{g}=o_{j}(1)
$$

This together with the Gauss-Bonnet formula (3) gives

$$
\int_{\Sigma} K_{\lambda} e^{2 u_{j}} d v_{g}=2 \pi \chi(\Sigma, \beta)+o_{j}(1)
$$

Inserting (22) into (19), we conclude

$$
\int_{\Sigma}\left(\left|\nabla_{g} u_{j}\right|^{2}+2 \kappa u_{j}\right) d v_{g}=c+2 \pi \chi(\Sigma, \beta)+o_{j}(1) .
$$

We now claim that $u_{j}$ is bounded in $L^{2}(\Sigma, g)$. Suppose not, there holds $\left\|u_{j}\right\|_{L^{2}(\Sigma, g)}$ $\rightarrow \infty$. We set $v_{j}=u_{j} /\left\|u_{j}\right\|_{L^{2}(\Sigma, g)}$. Note that

$$
\int_{\Sigma} \kappa \frac{u_{j}}{\left\|u_{j}\right\|_{L^{2}(\Sigma, g)}^{2}} d v_{g}=o_{j}(1) .
$$

This together with (23) tells us that

$$
\int_{\Sigma}\left|\nabla_{g} v_{j}\right|^{2} d v_{g}=o_{j}(1)
$$

Hence $v_{j}$ is bounded in $W^{1,2}(\Sigma, g)$ and (24) leads to $v_{j} \rightarrow \gamma$ in $W^{1,2}(\Sigma, g)$ for some constant $\gamma$. Since $\left\|v_{j}\right\|_{L^{2}(\Sigma, g)}=1$, we have $\gamma \neq 0$. It follows from (23) that

$$
\int_{\Sigma} \kappa v_{j} d v_{g} \leq o_{j}(1)
$$

Letting $j \rightarrow \infty$ in $(25)$, we obtain $2 \pi \chi(\Sigma, \beta) \gamma \leq 0$ by using the Gauss-Bonnet formula (3). Since $\chi(\Sigma, \beta)<0$ and $\gamma \neq 0$, we have $\gamma>0$. On the other hand, we conclude by (21) that

$$
\int_{\Omega^{\prime}}\left(\left|\nabla_{g} v_{j}^{+}\right|^{2}+v_{j}^{+2}\right) d v_{g}=o_{j}(1)
$$

which results in $\gamma=0$. This contradicts $\gamma>0$ and confirms our claim.

Since $u_{j}$ is bounded in $L^{2}(\Sigma, g)$, we have by $(23)$ that $u_{j}$ is bounded in $W^{1,2}(\Sigma, g)$. Up to a subsequence, we can assume $u_{j}$ converges to $u_{0}$ weakly in $W^{1,2}(\Sigma, g)$, strongly in $L^{s}(\Sigma, g)$ for any $s>1$. A Trudinger-Moser inequality for surfaces with conical 
singularities [28] implies that $e^{2 u_{j}}$ is bounded in $L^{s}(\Sigma, g)$ for any $s>1$. Hence $e^{u_{j}}$ converges to $e^{u_{0}}$ in $L^{s}(\Sigma, g)$ for any $s>1$. This together with (20) induces

$$
\int_{\Sigma}\left|\nabla_{g} u_{j}\right|^{2} d v_{g}=\int_{\Sigma}\left(-\kappa u_{0}+K_{\lambda} e^{2 u_{0}} u_{0}\right) d v_{g}+o_{j}(1)=\int_{\Sigma}\left|\nabla_{g} u_{0}\right|^{2} d v_{g}+o_{j}(1) .
$$

This implies that $u_{j} \rightarrow u_{0}$ in $W^{1,2}(\Sigma, g)$.

3.3. Upper and lower solutions principle. Let $f: \Sigma \times \mathbf{R} \rightarrow \mathbf{R}$ be a smooth function. We call $u \in W^{1,2}(\Sigma, g) \cap C^{2}(\Sigma \backslash \operatorname{supp} \beta) \cap C^{0}(\Sigma)$ an upper (lower) solution to the elliptic equation

$$
\Delta_{g} u+f(x, u)=0
$$

if $u$ satisfies $\Delta_{g} u+f(x, u) \geq(\leq) 0$ in the distributional sense on $\Sigma$ and point-wisely in $\Sigma \backslash \operatorname{supp} \beta$.

Lemma 6. (Upper and lower solutions principle) Suppose that $\psi, \varphi \in W^{1,2}(\Sigma, g) \cap$ $C^{2}(\Sigma \backslash \operatorname{supp} \beta) \cap C^{0}(\Sigma)$ are upper and lower solutions to (26) respectively, and that $\varphi \leq \psi$ on $\Sigma$. Then (26) has a solution $u \in W^{1,2}(\Sigma, g) \cap C^{2}(\Sigma \backslash \operatorname{supp} \beta) \cap C^{0}(\Sigma)$ with $\varphi \leq u \leq \psi$ on $\Sigma$.

Proof. We follow the lines of Kazdan and Warner [21]. Let $A$ be a constant such that $-A \leq \varphi \leq \psi \leq A$. Since $\Sigma$ is closed, one finds a sufficiently large constant $c$ such that $G(x, t)=c t+f(x, t)$ is increasing in $t \in[-A, A]$ for any fixed $x \in \Sigma$. Define an elliptic operator $L u=\Delta_{g} u+c u$ for $u \in W^{1,2}(\Sigma, g) \cap C^{2}(\Sigma \backslash \operatorname{supp} \beta) \cap C^{0}(\Sigma)$. Now we define

$$
\begin{array}{lll}
\varphi_{0}=\varphi, & \varphi_{j}=L^{-1}\left(G\left(x, \varphi_{j-1}\right)\right), & \forall j \geq 1 \\
\psi_{0}=\psi, & \psi_{j}=L^{-1}\left(G\left(x, \psi_{j-1}\right)\right), & \forall j \geq 1 .
\end{array}
$$

Here $L^{-1}: L^{2}(\Sigma, g) \rightarrow W^{1,2}(\Sigma, g)$ is well defined due to the Lax-Milgram theorem. This together with the definition of upper and lower solutions and the monotonicity of $G(x, t)$ with respect to $t$ leads to

$$
L \varphi \leq L \varphi_{1}=G(x, \varphi) \leq G(x, \psi)=L \psi_{1} \leq L \psi .
$$

Then the weak maximum principle (Lemma 3 ) implies that

$$
\varphi \leq \varphi_{1} \leq \psi_{1} \leq \psi
$$

By induction, we have

$$
\varphi \leq \varphi_{j-1} \leq \varphi_{j} \leq \psi_{j} \leq \psi_{j-1} \leq \psi, \quad j=1,2, \ldots
$$

Clearly we can assume that $\varphi_{j}$ converges to $u_{1}$ and $\psi_{j}$ converges to $u_{2}$ point-wisely. By elliptic estimates, one concludes that the above convergence is in $C_{\text {loc }}^{2}(\Sigma \backslash \operatorname{supp} \beta) \cap$ $C^{0}(\Sigma)$. Moreover, $v=u_{1}$ or $u_{2}$ is a distributional solution to $L v=G(x, v)$.

\section{Proof of Theorem 2}

In this section, we prove Theorem 2 by using variational method.

\subsection{Unique solution in the case $\boldsymbol{\lambda} \leq \mathbf{0}$.}

Proof of (i) of Theorem 2. Assume $\max _{\Sigma} K=0$ and $K \not \equiv 0$. If $\lambda<0$, this has been proved by Troyanov ([28], Theorem 1). We now consider the general case $\lambda \leq 0$. Let $E_{\lambda}$ be the functional defined as in (18), where $K_{\lambda}=K+\lambda$.

Claim 1. $E_{\lambda}$ is strict convex on $W^{1,2}(\Sigma, g)$. 
It suffices to prove that for any $u \in W^{1,2}(\Sigma, g)$, there exists some constant $C>0$ such that

$$
d^{2} E_{\lambda}(u)(h, h) \geq C\|h\|_{W^{1,2}(\Sigma, g)}^{2} \quad \forall h \in W^{1,2}(\Sigma, g) .
$$

Suppose not. There would be a function $u \in W^{1,2}(\Sigma, g)$ and a function sequence $\left(h_{j}\right) \subset W^{1,2}(\Sigma, g)$ such that $\left\|h_{j}\right\|_{W^{1,2}(\Sigma, g)}=1$ for all $j$ and $d^{2} E_{\lambda}(u)\left(h_{j}, h_{j}\right) \rightarrow 0$ as $j \rightarrow \infty$. One may assume up to a subsequence, $h_{j}$ converges to $h_{\infty}$ weakly in $W^{1,2}(\Sigma, g)$, strongly in $L^{p}(\Sigma, g)$ for any $p>1$, and almost everywhere in $\Sigma$. Since

$$
d^{2} E_{\lambda}(u)\left(h_{j}, h_{j}\right)=2 \int_{\Sigma}\left(\left|\nabla_{g} h_{j}\right|^{2}-2 K_{\lambda} e^{2 u} h_{j}^{2}\right) d v_{g}
$$

and $K_{\lambda} \leq 0$, we conclude $\int_{\Sigma}\left|\nabla_{g} h_{j}\right|^{2} d v_{g} \rightarrow 0$ and $\int_{\Sigma} K_{\lambda} e^{2 u} h_{j}^{2} d v_{g} \rightarrow 0$, which leads to $h_{\infty} \equiv C_{0}$ for some constant $C_{0}$, and further

$$
C_{0}^{2} \int_{\Sigma} K_{\lambda} e^{2 u} d v_{g}=\int_{\Sigma} K_{\lambda} e^{2 u} h_{\infty}^{2} d v_{g}=\lim _{j \rightarrow \infty} \int_{\Sigma} K_{\lambda} e^{2 u} h_{j}^{2} d v_{g}=0 .
$$

Clearly $\int_{\Sigma} K_{\lambda} e^{2 u} d v_{g}<0$, and thus $C_{0}=0$. This contradicts

$$
\left\|h_{\infty}\right\|_{L^{2}(\Sigma, g)}=\lim _{j \rightarrow \infty}\left\|h_{j}\right\|_{L^{2}(\Sigma, g)}=1 .
$$

Hence (27) holds.

Claim 2. $E_{\lambda}$ is coercive.

Since for any $\epsilon>0$, there exists a constant $C(\epsilon)$ such that $\int_{\Sigma} \kappa u d v_{g} \leq \epsilon\|u\|_{W^{1,2}(\Sigma, g)}^{2}$ $+C(\epsilon)$, it suffices to find some constant $C>0$ such that for all $u \in W^{1,2}(\Sigma, g)$, there holds

$$
\int_{\Sigma}\left(\left|\nabla_{g} u\right|^{2}-K_{\lambda} e^{2 u}\right) d v_{g} \geq C\|u\|_{W^{1,2}(\Sigma, g)}^{2}
$$

Suppose not. There would exist a sequence of functions $\left(u_{j}\right)$ satisfying

$$
\int_{\Sigma}\left(\left|\nabla_{g} u_{j}\right|^{2}+u_{j}^{2}\right) d v_{g}=1, \quad \int_{\Sigma}\left(\left|\nabla_{g} u_{j}\right|^{2}-K_{\lambda} e^{2 u_{j}}\right) d v_{g}=o_{j}(1) .
$$

It follows that up to a subsequence, $u_{j}$ converges to $u^{*}$ weakly in $W^{1,2}(\Sigma, g)$ and strongly in $L^{p}(\Sigma, g)$ for any $p>1$. One easily see that

$$
0<\int_{\Sigma}\left(\left|\nabla_{g} u^{*}\right|^{2}-K_{\lambda} e^{2 u^{*}}\right) d v_{g} \leq \lim _{j \rightarrow \infty} \int_{\Sigma}\left(\left|\nabla_{g} u_{j}\right|^{2}-K_{\lambda} e^{2 u_{j}}\right) d v_{g}=0,
$$

which is impossible. Hence (28) holds.

In view of Claims 1 and 2 , a direct method of variation shows $\inf _{u \in W^{1,2}(\Sigma, g)} E_{\lambda}(u)$ can be attained by some $u_{0} \in W^{1,2}(\Sigma, g)$ and $u_{0}$ is the unique critical point of $E_{\lambda}$.

4.2. Existence of $\lambda^{*}$. When $\lambda=0$, the equation (7) becomes

$$
\Delta_{g} u+\kappa-K e^{2 u}=0 \quad \text { on } \Sigma \text {. }
$$

Let $u$ be a solution of (29). The linearized equation of (29) at $u$ reads $\Delta_{g} v-2 K e^{2 u} v=$ 0 , which has a unique solution $v \equiv 0$. By the implicit theorem, there is a sufficiently small $s>0$ such that for any $\lambda \in(0, s)$, the equation (7) has a solution. Define

$$
\lambda^{*}=\sup \{s \text { : the equation }(7) \text { has a solution for any } \lambda \in(0, s)\} \text {. }
$$


One can see that $\lambda^{*} \leq-\min _{\Sigma} K$. For otherwise $K_{\lambda}>0$ for some $\lambda<\lambda^{*}$. Integrating (7), we obtain

$$
0>2 \pi \chi(\Sigma, \boldsymbol{\beta})=\int_{\Sigma} \kappa d v_{g}=\int_{\Sigma} K_{\lambda} e^{2 u} d v_{g} \geq 0
$$

which is impossible. In conclusion, we have $0<\lambda^{*} \leq-\min _{\Sigma} K$. Further analysis (Subsection 4.4, Claim 2) implies that $\lambda^{*}<-\min _{\Sigma} K$.

\subsection{Multiplicity of solutions for $0<\lambda<\lambda^{*}$.}

Proof of (ii) of Theorem 2. Fix $\lambda, 0<\lambda<\lambda^{*}$. We shall seek two different solutions of (7), one is a strict local minimum of the functional $E_{\lambda}$, the other is of the mountain-pass type. The proof will be divided into several steps below.

Step 1. Existence of upper and lower solutions.

Take $\lambda_{1}$ with $\lambda<\lambda_{1}<\lambda^{*}$. Let $u_{\lambda_{1}} \in W^{1,2}(\Sigma, g) \cap C^{2}(\Sigma \backslash \operatorname{supp} \beta) \cap C^{0}(\Sigma)$ be a solution of (7) at $\lambda_{1}$. Set $\psi=u_{\lambda_{1}}$. One can see that $\psi$ is a strict upper solution of (7), namely

$$
\Delta_{g} \psi+\kappa-K_{\lambda} e^{2 \psi}>0
$$

Clearly the equation

$$
\Delta_{g} \eta=-\kappa+\frac{1}{\operatorname{Vol}_{g}(\Sigma)} \int_{\Sigma} \kappa d v_{g}
$$

has a distributional solution $\eta \in W^{1,2}(\Sigma, g) \cap C^{2}(\Sigma \backslash \operatorname{supp} \beta) \cap C^{0}(\Sigma)$. Let $\varphi=\eta-s$, where $s$ is a positive constant. Obviously $\varphi<\psi$ on $\Sigma$ for sufficiently large $s$. Since $\int_{\Sigma} \kappa d v_{g}=2 \pi \chi(\Sigma, \boldsymbol{\beta})<0$, we have

$$
\Delta_{g} \varphi+\kappa-K_{\lambda} e^{2 \varphi}=\frac{1}{\operatorname{Vol}_{g}(\Sigma)} \int_{\Sigma} \kappa d v_{g}-K_{\lambda} e^{2 \eta-2 s}<0,
$$

provided that $s$ is chosen sufficiently large. Thus $\varphi$ is a strict lower solution of (7).

Step 2. The first solution of (7) can be chosen as a strict local minimum of $E_{\lambda}$.

Let $f_{\lambda}(x, t)=c t-\kappa+K_{\lambda} e^{2 t}$. Fix a sufficiently large positive constant $c$ such that $f_{\lambda}(x, t)$ is increasing in $t \in[-A, A]$, where $A$ is a constant such that $-A \leq \varphi<$ $\psi \leq A$. Let $F_{\lambda}(x, u)=\int_{0}^{u} f_{\lambda}(x, t) d t$. It is easy to see that

$$
E_{\lambda}(u)=\int_{\Sigma}\left|\nabla_{g} u\right|^{2} d v_{g}+c \int_{\Sigma} u^{2} d v_{g}-2 \int_{\Sigma} F_{\lambda}(x, u) d v_{g}-\int_{\Sigma} K_{\lambda} d v_{g} .
$$

Define a function

$$
\hat{f}_{\lambda}(x, t)= \begin{cases}f_{\lambda}(x, \psi(x)) & \text { when } t>\psi(x) \\ f_{\lambda}(x, t) & \text { when } \varphi(x) \leq t \leq \psi(x), \\ f_{\lambda}(x, \varphi(x)) & \text { when } t<\varphi(x)\end{cases}
$$

and a functional

$$
\hat{E}_{\lambda}(u)=\int_{\Sigma}\left(\left|\nabla_{g} u\right|^{2}+c u^{2}\right) d v_{g}-2 \int_{\Sigma} \hat{F}_{\lambda}(x, u) d v_{g}-\int_{\Sigma} K_{\lambda} d v_{g}
$$

where $\hat{F}_{\lambda}(x, t)=\int_{0}^{t} \hat{f}_{\lambda}(x, s) d s$. Obviously $\hat{E}_{\lambda}$ is bounded from below on $W^{1,2}(\Sigma, g)$. Denote

$$
a=\inf _{u \in W^{1,2}(\Sigma, g)} \hat{E}_{\lambda}(u) .
$$


Taking a function sequence $\left(u_{j}\right) \subset W^{1,2}(\Sigma, g)$ such that $\hat{E}_{\lambda}\left(u_{j}\right) \rightarrow a$ as $j \rightarrow \infty$. It follows that $u_{j}$ is bounded in $W^{1,2}(\Sigma, g)$, and thus up to a subesequence the Sobolev embedding and the Trudinger-Moser inequality lead to $u_{j}$ converges to some $u_{\lambda}$ weakly in $W^{1,2}(\Sigma, g)$, strongly in $L^{q}(\Sigma, g)$ for any $q>1$, almost everywhere in $\Sigma$, and $e^{2 u_{j}}$ converges to $e^{2 u_{\lambda}}$ in $L^{1}(\Sigma, g)$. Hence $\hat{E}_{\lambda}\left(u_{\lambda}\right) \leq a$. Then by the definition of $a$, we conclude

$$
\hat{E}_{\lambda}\left(u_{\lambda}\right)=\inf _{u \in W^{1,2}(\Sigma, g)} \hat{E}_{\lambda}(u)
$$

As a consequence $u_{\lambda}$ satisfies the Euler-Lagrange equation

$$
\Delta_{g} u_{\lambda}+c u_{\lambda}=\hat{f}_{\lambda}\left(x, u_{\lambda}\right)
$$

in the distributional sense. By elliptic estimates, one has $u_{\lambda} \in C^{2}(\Sigma \backslash \operatorname{supp} \beta) \cap C^{0}(\Sigma)$.

Noting that $f(x, t)$ is increasing with respect to $t \in[-A, A]$, we have

$$
\Delta_{g} \varphi(x)+c \varphi(x) \leq f_{\lambda}(x, \varphi(x)) \leq \hat{f}_{\lambda}\left(x, u_{\lambda}(x)\right) \leq f_{\lambda}(x, \psi(x)) \leq \Delta_{g} \psi(x)+c \psi(x)
$$

in the distributional sense. In view of (31), (33) and (34), one concludes by the strong maximum principle (Lemma 4 ) that

$$
\varphi(x)<u_{\lambda}(x)<\psi(x), \quad \forall x \in \Sigma .
$$

Obviously $\hat{E}_{\lambda}(u)=E_{\lambda}(u)$ for all $u \in W^{1,2}(\Sigma, g)$ with $\varphi \leq u \leq \psi$. For any $h \in C^{1}(\Sigma)$, we define a function $\zeta(t)=E\left(u_{\lambda}+t h\right), t \in \mathbf{R}$. In view of (35), there holds $\varphi \leq$ $u_{\lambda}+t h \leq \psi$ and thus $\hat{E}_{\lambda}\left(u_{\lambda}+t h\right)=E_{\lambda}\left(u_{\lambda}+t h\right)$, provided that $|t|$ is sufficiently small. Since $u_{\lambda}$ is a minimum of $\hat{E}_{\lambda}$ on $W^{1,2}(\Sigma, g)$, we have $\zeta^{\prime}(0)=d E_{\lambda}\left(u_{\lambda}\right)(h)=0$ and $\zeta^{\prime \prime}(0)=d^{2} E_{\lambda}\left(u_{\lambda}\right)(h, h) \geq 0$. Therefore we have

$$
\begin{array}{r}
\int_{\Sigma}\left(\nabla_{g} u_{\lambda} \nabla_{g} h+\kappa h-K_{\lambda} e^{2 u_{\lambda}} h\right) d v_{g}=0, \quad \forall h \in C^{1}(\Sigma), \\
\int_{\Sigma}\left(\left|\nabla_{g} h\right|^{2}-2 K_{\lambda} e^{2 u_{\lambda}} h^{2}\right) d v_{g} \geq 0, \quad \forall h \in C^{1}(\Sigma) .
\end{array}
$$

Since $C^{1}(\Sigma)$ is dense in $W^{1,2}(\Sigma, g)$, (36) and (37) still hold for all $h \in W^{1,2}(\Sigma, g)$. We further prove that there exists a positive constant $C$ such that

$$
d^{2} E_{\lambda}\left(u_{\lambda}\right)(h, h) \geq C\|h\|_{W^{1,2}(\Sigma, g)}^{2}, \quad \forall h \in W^{1,2}(\Sigma, g) .
$$

For the proof of (38), we adapt an argument of Borer, Galimberti, and Struwe ([5], Section 2). Since $d^{2} E_{\lambda}\left(u_{\lambda}\right)(h, h) \geq 0$ for all $h \in W^{1,2}(\Sigma, g)$, we have

$$
\Lambda:=\inf _{\|h\|_{W^{1,2}(\Sigma, g)}=1} d^{2} E_{\lambda}\left(u_{\lambda}\right)(h, h) \geq 0 .
$$

Suppose $\Lambda=0$. We claim that there exists some $h$ with $\|h\|_{W^{1,2}(\Sigma, g)}=1$ such that $d^{2} E_{\lambda}\left(u_{\lambda}\right)(h, h)=0$. To see this, we let $h_{j}$ satisfy $\left\|h_{j}\right\|_{W^{1,2}(\Sigma, g)}=1$ and $d^{2} E_{\lambda}\left(u_{\lambda}\right)\left(h_{j}, h_{j}\right) \rightarrow 0$ as $j \rightarrow \infty$. Up to a subsequence, we can assume $h_{j}$ converges to some $h$ weakly in $W^{1,2}(\Sigma, g)$, strongly in $L^{q}(\Sigma, g)$ for all $q>1$, and almost everywhere in $\Sigma$. It follows that

$$
\lim _{j \rightarrow \infty} \int_{\Sigma}\left|\nabla_{g} h_{j}\right|^{2} d v_{g}=\int_{\Sigma} 2 K_{\lambda} e^{2 u_{\lambda}} h^{2} d v_{g} \leq \int_{\Sigma}\left|\nabla_{g} h\right|^{2} d v_{g} .
$$

This leads to $h_{j} \rightarrow h$ in $W^{1,2}(\Sigma, g)$ as $j \rightarrow \infty$, and confirms our claim. Moreover, since the functional $v \mapsto d^{2} E_{\lambda}\left(u_{\lambda}\right)(v, v)$ attains its minimum at $v=h$, it follows 
that $d^{2} E_{\lambda}\left(u_{\lambda}\right)(h, w)=0$ for all $w \in W^{1,2}(\Sigma, g)$; that is, $h$ is a weak solution of the equation

$$
\Delta_{g} h=2 K_{\lambda} e^{2 u_{\lambda}} h
$$

Note that $h$ is not a constant. For otherwise (39) yields

$$
0>2 \pi \chi(\Sigma, \beta)=\int_{\Sigma} K_{\lambda} e^{2 u_{\lambda}} d v_{g}=0
$$

which is impossible. Multiplying (39) by $h^{3}$, we get

$$
d^{4} E_{\lambda}\left(u_{\lambda}\right)(h, h, h, h)=-16 \int_{\Sigma} K_{\lambda} e^{2 u_{\lambda}} h^{4} d v_{g}=-24 \int_{\Sigma} h^{2}\left|\nabla_{g} h\right|^{2} d v_{g}<0 .
$$

Now we expand $E_{\lambda}$ around $u_{\lambda}$. Since $d^{2} E_{\lambda}\left(u_{\lambda}+t h\right)(h, h)$ attains its minimum at $t=0$, we have $d^{3} E_{\lambda}\left(u_{\lambda}\right)(h, h, h)=0$, which together with the facts $d E_{\lambda}\left(u_{\lambda}\right)=0$ and $d^{2} E_{\lambda}\left(u_{\lambda}\right)(h, h)=0$ leads to

$$
E_{\lambda}\left(u_{\lambda}+\epsilon h\right)=E_{\lambda}\left(u_{\lambda}\right)+\frac{\epsilon^{4}}{24} d^{4} E_{\lambda}\left(u_{\lambda}\right)(h, h, h, h)+O\left(\epsilon^{5}\right)<E_{\lambda}\left(u_{\lambda}\right)
$$

for small $\epsilon>0$. Applying elliptic estimates to (39), we have $h \in C^{0}(\Sigma)$. Then there exists $\epsilon_{0}>0$ such that if $0<\epsilon<\epsilon_{0}$, then $\varphi \leq u_{\lambda}+\epsilon h \leq \psi$ on $\Sigma$, and thus by (40),

$$
\hat{E}_{\lambda}\left(u_{\lambda}+\epsilon h\right)=E_{\lambda}\left(u_{\lambda}+\epsilon h\right)<E_{\lambda}\left(u_{\lambda}\right)=\hat{E}_{\lambda}\left(u_{\lambda}\right),
$$

contradicting the fact that $u_{\lambda}$ is the minimum of $\hat{E}_{\lambda}$. Therefore $\Lambda>0$ and (38) follows immediately. As a consequence, $u_{\lambda}$ is a strict local minimum of $E_{\lambda}$ on $W^{1,2}(\Sigma, g)$.

Step 3. The second solution of (7) can be achieved by a mountain pass theorem.

Let $u_{\lambda}$ be as in Step 2. Since $u_{\lambda}$ is a strict local minimum of $E_{\lambda}$ on $W^{1,2}(\Sigma, g)$, there would exist a sufficiently small $r>0$ such that

$$
\inf _{\left\|u-u_{\lambda}\right\|_{W^{1,2}(\Sigma, g)}=r} E_{\lambda}(u)>E_{\lambda}\left(u_{\lambda}\right) .
$$

Moreover, a calculation of Ding and Liu ([16], Page 1061) shows for any $\lambda>0, E_{\lambda}$ has no lower bound on $W^{1,2}(\Sigma, g)$. In particular, there exists some $v \in W^{1,2}(\Sigma, g)$ verifying that

$$
E_{\lambda}(v)<E_{\lambda}\left(u_{\lambda}\right), \quad\left\|v-u_{\lambda}\right\|_{W^{1,2}(\Sigma, g)}>r .
$$

Combining (41), (42) and Lemma 5 , we obtain by using the mountain-pass theorem due to Ambrosetti and Rabinowitz [2] that the mini-max value

$$
c=\min _{\gamma \in \Gamma} \max _{u \in \gamma} E_{\lambda}(u)
$$

is a critical value of $E_{\lambda}$, where $\Gamma=\left\{\gamma \in \mathcal{C}\left([0,1], W^{1,2}(\Sigma, g)\right): \gamma(0)=u_{\lambda}, \gamma(1)=v\right\}$. Equivalently there exists some $u^{\lambda} \in W^{1,2}(\Sigma, g)$ satisfying $E_{\lambda}\left(u^{\lambda}\right)=c$ and $d E_{\lambda}\left(u^{\lambda}\right)=$ 0 . Thus $u^{\lambda}$ is a solution of the equation (7) and $u^{\lambda} \neq u_{\lambda}$. Finally, elliptic estimates imply that $u^{\lambda} \in C^{2}(\Sigma \backslash \operatorname{supp} \beta) \cap C^{0}(\Sigma)$.

\subsection{Solvability of $(7)$ at $\lambda^{*}$.}

Proof of (iii) of Theorem 2. For any $\lambda, 0<\lambda<\lambda^{*}$, we let $u_{\lambda}$ be the local minimum of $E_{\lambda}$ obtained in the previous subsection. In particular, $u_{\lambda}$ is a solution of $(7)$ and

$$
\int_{\Sigma}\left(\left|\nabla_{g} \phi\right|^{2}-2 K_{\lambda} e^{2 u_{\lambda}} \phi^{2}\right) d v_{g} \geq 0, \quad \forall \phi \in W^{1,2}(\Sigma, g)
$$

The remaining part of the proof will be divided into several claims as below. 
Claim 1. There exists some constant $C$ such that $u_{\lambda} \geq-C$ on $\Sigma$ uniformly in $\lambda \in\left(0, \lambda^{*}\right)$.

To see this, we let $\eta$ satisfy (32) and $\varphi_{s}=\eta-s$ for $s>0$. The analog of (33) reads

$$
\Delta_{g} \varphi_{s}+\kappa-K e^{2 \varphi_{s}}<0
$$

with $s$ chosen sufficiently large, say $s \geq s_{0}$. Equivalently $\varphi_{s}$ is a lower solution of (7) at $\lambda=0$, provided that $s \geq s_{0}$. Clearly $\varphi_{s}$ is also a strict lower solution of (7) at $\lambda \in\left(0, \lambda^{*}\right)$ for any $s \geq s_{0}$. We now prove that $u_{\lambda} \geq \varphi_{s_{0}}$, and consequently claim 1 holds. Suppose not, by varying $s \in\left[s_{0}, \infty\right)$, we find that for some $s$ there holds $u_{\lambda} \geq \varphi_{s}$ on $\Sigma$, and $u_{\lambda}\left(x_{0}\right)=\varphi_{s}\left(x_{0}\right)$ for some $x_{0} \in \Sigma$. Then the strong maximum principle (Lemma 4) implies that $u_{\lambda} \equiv \varphi_{s}$ on $\Sigma$, which is impossible.

Claim 2. Let $\Sigma_{\lambda^{*}}^{-}=\left\{x \in \Sigma: K_{\lambda^{*}}(x)<0\right\}$. Then $\Sigma_{\lambda^{*}}^{-} \neq \varnothing$.

Suppose $K_{\lambda^{*}} \geq 0$. Let $\widetilde{g}=e^{2 v} g$ be a metric with constant Gaussian curvature -1 , where $v$ is a solution of $\Delta_{g} v+\kappa+e^{2 v}=0$. In view of $(i)$ of Theorem 2, such a function $v$ uniquely exists. Let $w_{\lambda}=u_{\lambda}-v$. Noting that $\Delta_{g}=e^{2 v} \Delta_{\widetilde{g}}$, we have

$$
\Delta_{\widetilde{g}} w_{\lambda}-1-K_{\lambda} e^{2 w_{\lambda}}=0
$$

Multiplying the above equation by $e^{-2 w_{\lambda}}$ and integrating by parts, one has

$$
\int_{\Sigma} K_{\lambda} d v_{\widetilde{g}}=-\int_{\Sigma} e^{-2 w_{\lambda}} d v_{\widetilde{g}}-2 \int_{\Sigma}\left|\nabla_{\widetilde{g}} w_{\lambda}\right|^{2} e^{-2 w_{\lambda}} d v_{\widetilde{g}} \leq 0 .
$$

Hence

$$
\int_{\Sigma} K_{\lambda^{*}} d v_{\widetilde{g}}=\lim _{\lambda \rightarrow \lambda^{*}} \int_{\Sigma} K_{\lambda} d v_{\widetilde{g}} \leq 0
$$

This together with $K_{\lambda^{*}} \geq 0$ leads to $K_{\lambda^{*}} \equiv 0$, which contradicts the assumption that $K_{\lambda^{*}}$ is not a constant.

Claim 3. Let $\Omega$ and $\Omega^{\prime}$ are two domains in $\Sigma$ such that $\Omega^{\prime} \subset \subset \Omega \subset \subset \Sigma_{\lambda^{*}}^{-} \backslash \operatorname{supp} \beta$. Then $u_{\lambda}^{+}$is bounded in $W^{1,2}\left(\Omega^{\prime}, g\right)$ with respect to $\lambda \in\left(0, \lambda^{*}\right)$.

Note that $K: \Sigma \rightarrow \mathbf{R}$ is Hölder continuous. If $\lambda \in\left(0, \lambda^{*}\right)$, then

$$
\sup _{\Omega} K_{\lambda} \leq \sup _{\Omega} K_{\lambda^{*}} \leq-\epsilon
$$

for some $\epsilon>0$ depending only on $K, \lambda^{*}$ and $\Omega$. Similar to the proof of (21), we conclude Claim 3.

Claim 4. The equation (7) is solvable at $\lambda^{*}$.

Having Claims 1-3 in hand and arguing as Ding and Liu did in the proof of ([16], (c) of the main theorem), we conclude that both $e^{2 u_{\lambda}}$ and $u_{\lambda}$ are bounded in $L^{q}(\Sigma, g)$ for all $q>1$. By elliptic estimates, we have up to a subsequence, $u_{\lambda}$ converges to some $u$ in $W^{1,2}(\Sigma, g)$, where $u$ is a solution of

$$
\Delta_{g} u+\kappa-K_{\lambda^{*}} e^{2 u}=0 .
$$

By elliptic estimates, $u \in C^{2}(\Sigma \backslash \operatorname{supp} \beta) \cap C^{0}(\Sigma)$. This gives the desired result.

\subsection{The equation (7) has no distributional solution when $\boldsymbol{\lambda}>\boldsymbol{\lambda}^{*}$.}

Proof of (iv) of Theorem 2. Suppose (7) has a solution $u_{\lambda_{1}}$ at some $\lambda_{1}>\lambda^{*}$. Then for any $\lambda, 0<\lambda<\lambda_{1}, u_{\lambda_{1}}$ is an upper solution of (7). Similar to (33), we can easily construct a lower solution $\varphi$ of (7) such that $\varphi \leq u_{\lambda_{1}}$. In view of the upper and lower solutions principle (Lemma 6 ), there would exist a solution of (7), which contradicts (30). 
Acknowledgements. This work is supported by National Science Foundation of China (Grant Nos. 11171347, 11471014 and 11401575).

\section{References}

[1] Adimurthi, and Y. YAng: An interpolation of Hardy inequality and Trudinger-Moser inequality in $\mathbf{R}^{N}$ and its applications. - Int. Math. Res. Not. IMRN 13, 2010, 2394-2426.

[2] Ambrosetti, A., and P. Rabinowitz: Dual variational methods in critical point theory and applications. J. Funct. Anal. 14, 1973, 349-381.

[3] Aubin, T., and S. Bismuth: Courbure scalaire prescrite sur les variétés riemanniennes compactes dans le cas négatif. - J. Funct. Anal. 143, 1997, 529-541.

[4] Bismuth, S.: Prescribed scalar curvature on a $C^{\infty}$ compact Riemannian manifold of dimension two. - Bull. Sci. Math. 124, 2000, 239-248.

[5] Borer, F., L. Galimberti, and M. Struwe: "Large" conformal metrics of prescribing Gauss curvature on surfaces of high genus. - Comment. Math. Helv. 90, 2015, 407-428.

[6] Chang, A., and P. Yang: Prescribing Gaussian curvatures on $S^{2}$. - Acta Math. 159, 1987, 214-259.

[7] Chang, A., and P. YAng: Conformal deformation of metrics on $S^{2}$. - J. Differential Geom. 27, 1988, 259-296.

[8] Chang, K., and J. Liu: On Nirenberg's problem. - Internat. J. Math. 4, 1993), 35-58.

[9] Chen, W., and W. Ding: Scalar curvatures on $S^{2}$. - Trans. Amer. Math. Soc. 303, 1987, $365-382$.

[10] Chen, W., and C. Li: Prescribing Gaussian curvatures on surfaces with conical singularities. - J. Geom. Anal. 1, 1991, 359-372.

[11] Chen, W., and C. Li: Gaussian curvature on singular surfaces. - J. Geom. Anal. 3, 1993, $315-334$.

[12] Chen, C., and C. Lin: Mean field equation of Liouville type with singular data: topological degree. - Comm. Pure Appl. Math. 68, 2015, 887-947.

[13] Csato, G., and P. Roy: Extremal functions for the singular Moser-Trudinger inequality in 2 dimensions. - Calc. Var. Partial Differential Equations 54, 2015, 2341-2366.

[14] De Marchis, F., and R. López-Soriano: Existence and non existence results for the singular Nirenberg problem- - Calc. Var. Partial Differential Equations 55, Art. 36, 2016, 1-35.

[15] DEL Pino, M., and C. Román: Large conformal metrics with prescribed sign-changing Gauss curvature. - Calc. Var. Partial Differential Equations 54, 2015, 763-789.

[16] Ding, W., and J. LiU: A note on the problem of prescribing Gaussian curvature on surfaces. - Trans. Amer. Math. Soc. 347, 1995, 1059-1066.

[17] Eremenko, A.: Metrics of positive curvature with conical singularities on the sphere. - Proc. Amer. Math. Soc. 132, 2004, 3349-3355.

[18] Galimberti, L.: Compactness issues and bubbling phenomena for the prescribed Gaussian curvature equation on the torus. - Calc. Var. Partial Differential Equations 54, 2015, 2483-2501.

[19] Gilbarg, D., and N. Trudinger: Elliptic partial differential equations of second order. Springer, 2001.

[20] Iula, S., and G. Mancini: Extremal functions for singular Moser-Trudinger embeddings. Nonlinear Anal. 156, 2017, 215-248.

[21] Kazdan, J., and F. WARner: Curvature functions for compact 2-manifolds. - Ann. Math. 99, $1974,14-47$.

[22] Li, X., and Y. YANG: Extremal functions for singular Trudinger-Moser inequalities in the entire Euclidean space. - J. Differential Equations 264, 2018, 4901-4943. 
[23] Malchiodi, A., and D. Ruiz: Now improved Moser-Trudinger inequalities and singular Liouville equations on compact surfaces. - Geom. Funct. Anal. 21, 2011, 1196-1217.

[24] Moser, J.: On a nonlinear problem in differential geometry. - Dynamical systems, Academic Press, New York, 1973.

[25] Nguyen, V.: Extremal functions for the Moser-Trudinger inequality of Adimurthi-Druet type in $W^{1, N}\left(\mathbf{R}^{N}\right)$. - arXiv: 1702.07970 .

[26] Struwe, M.: Critical points of embeddings of $H_{0}^{1, n}$ into Orlicz spaces. - Ann. Inst. H. Poincaré Anal. Non Linéaire 5, 1988, 19-64.

[27] Struwe, M.: The existence of surfaces of constant mean curvature with free boundaries. Acta Math. 160, 1988, 19-64.

[28] Troyanov, M.: Prescribing curvature on compact surfaces with conical singularities. - Trans. Amer. Math. Soc. 324, 1991, 251-256.

[29] YANG, Y.: Extremal functions for Trudinger-Moser inequalities of Adimurthi-Druet type in dimension two. - J. Differential Equations 258, 2015, 3161-3193.

[30] YANG, Y., and X. ZHU: Blow-up analysis concerning singular Trudinger-Moser inequalities in dimension two. - J. Funct. Anal. 272, 2017, 3347-3374.

Received 2 May $2017 \bullet$ Accepted 8 August 2018 